\title{
Transport in molecular states language: Generalized quantum master equation approach
}

\author{
Massimiliano Esposito* and Michael Galperin \\ Department of Chemistry and Biochemistry, University of California San Diego, La Jolla, California 92093, USA
}

(Received 24 November 2008; revised manuscript received 16 February 2009; published 6 May 2009)

\begin{abstract}
A simple scheme, capable of treating transport in molecular junctions in the language of many-body states, is presented. By introducing an ansatz in Liouville space, similar to the generalized Kadanoff-Baym approximation, a quantum master equation (QME)-like expression is derived starting from the exact equation of motion for Hubbard operators. Using an effective Liouville space propagation, a dressing similar to the standard diagrammatic one is proposed. The scheme is compared to the standard QME approach and its applicability to transport calculations is discussed.
\end{abstract}

DOI: 10.1103/PhysRevB.79.205303

PACS number(s): 73.63.Kv, 73.23.Hk, 85.65.+h, 85.35.Be

\section{INTRODUCTION}

Quantum transport in nanoscale systems is at the forefront of research in many different fields. In particular, the progress of the experimental capabilities in the field of molecular electronics brings many new theoretical challenges. ${ }^{1}$ Resonant transport with strong on-the-bridge interactions is one of them. It is probably the most important regime for possible future applications such as logic and memory molecular devices. Unlike usual mesoscopic systems, molecular electronic (and vibrational) structure may be very sensitive to reduction/oxidation. A possible way to account for the consequences of strong on-the-molecule interactions is to apply the standard diagrammatic techniques, e.g., $G W$ approximation, ${ }^{2-5}$ to nonequilibrium systems. Another approach is to describe resonant transport in molecular junctions in the language of molecular states (states of the isolated molecule), rather than in the language of (effective) single-particle orbitals as is usually done in the molecular electronics community. Recent experiments on simultaneous measurements of current and optical response of molecular junctions ${ }^{6}$ make the need for such a formulation even more pronounced, since molecular states is the natural language of the equilibrium molecular spectroscopy. Besides, a formulation of transport based on molecular states potentially allows the incorporation of standard quantum chemistry molecular structure simulations as an input for the transport calculations.

Attempts have been made in the past to formulate a manybody state description of transport in molecular systems. Among these approaches, one can mention scattering theory, ${ }^{7}$ rate equations, ${ }^{8-14}$ quantum master equations (QMEs), ${ }^{15-21}$ and the nonequilibrium Green's-function (GF)based schemes. ${ }^{22-24}$ Each of these has its own limitations. When applied to transport problems, scattering theory disregards important many-body effects. This may lead to erroneous predictions. ${ }^{25,26}$ Standard scattering theory formulations also miss some important effects such as effective attractive electron-electron interactions via phonons (bipolaron formation), energy exchange (heating and cooling effects) between successive tunneling events, and target distortion due to quasibound states. Rate equations (or generalized master equation) approaches are often used to describe hopping transport, i.e., situations when correlations in the system (both in space and time) die much faster than electron transfer time (determined by contact/molecule coupling). Besides, they become inadequate to describe off-resonant tunneling (superexchange). QMEs schemes are often restricted to weak contact/molecule coupling and thus miss the broadening of the molecular states as well as coherences which are responsible, for example, for the elastic channel renormalization at the inelastic threshold (see also discussion below). Nonequilibrium Green function (NEGF) approach in the language of molecular states is among the most advanced methods for treating nonequilibrium molecular systems. ${ }^{24}$ However, two important drawbacks of this approach are its complicated character and the absence of proper commutation relations for the Hubbard operators. The first means that applicability of the method is limited to simple cases only. The second means that it may lead to unphysical results (e.g., nonHermiticity of the reduced density matrix) at an approximate level of treatment. ${ }^{27,28}$

The goal of this paper is to propose an approximate treatment of transport in molecular junctions in the language of molecular states and to explore the connection between GF and density matrix (DM) approaches to transport in a manner similar to our previous consideration. ${ }^{29} \mathrm{We}$ start from exact NEGF consideration and systematically derive QME by carefully pointing out the approximations involved in the derivation. In the limit of weak system-bath coupling, we recover the standard QME result. We note that the Kondo effect is beyond the scope of our current consideration.

Let us make a few comments with regard to other works. An approach similar to ours, but in terms of single-particle orbitals, was used in Ref. 30. In the absence of on-themolecule interactions and within effective second order in system-bath coupling (see discussion below) our consideration reduces to that of Ref. 30. The issue of deriving QMEs which display broadening has been recently addressed also in Ref. 18. These results rely on the Markovian approximation and on perturbative treatment (beyond second order) in the contact/molecule coupling. Our central result [Eq. (35)] is formally nonperturbative and non-Markovian. It is only after the Markovian approximation for effective evolution operator $[\mathrm{Eq}$. (36)] that our QME becomes an effective second-order scheme in system-bath coupling. Another approach, Ref. 31, tries to mimic standard NEGF when one works in the many-body states language (Fock space). Its main results, an analog of the Dyson and the Keldysh equa- 
tions of standard NEGF approach, are correct to the effective second order in system-bath interaction. The main approximation in this approach is to neglect the correlations between the "self-energy" and the GF.

We work with Hubbard operators since they are the natural objects capable of describing excitations in the molecule as transitions between many-body molecular states. We start by showing how the exact equations of motion for the Hubbard operators can be reduced to a QME form by introducing an ansatz in Liouville space similar to the generalized Kadanoff-Baym approximation ${ }^{32}$ in Hilbert space. Then, we identify the diagrams on the Keldysh contour corresponding to the processes described by QME and, in the spirit of GF diagrammatic techniques, we dress these diagrams using an effective Liouville space dynamics. The plan of the paper is as follows. In Sec. II, we introduce the molecular junction model and present the exact equation-of-motion for the Hubbard operator. In Sec. III, we introduce the ansatz in the Liouville space and use it to derive a closed QME. We then discuss the dressing procedure. In Sec. IV, we present some analytical results for a simple resonant level model and discuss the general procedure for numerical implementation of our scheme. We end the section by presenting several numerical results for different models. Conclusions are drawn in Sec. V.

\section{MODEL AND ITS EXACT DYNAMICS}

We consider a molecular junction which consists of two contacts $(L$ and $R$ ) coupled via the molecule $(M)$. The contacts are assumed to be reservoirs of free electrons each at its own equilibrium. All the nonequilibrium processes take place on the molecule. The Hamiltonian of the system is

$$
\hat{H}=\hat{H}_{L}+\hat{H}_{M}+\hat{H}_{R}+\hat{V} \equiv \hat{H}_{0}+\hat{V},
$$

where $\hat{H}_{M}$ is the full molecular Hamiltonian (i.e., the Hamiltonian of isolated molecule with all on-the-molecule interactions included), $\hat{V}$ is the molecule-contacts coupling

$$
\hat{V}=\sum_{m \in M, k \in\{L, R\}}\left(V_{k m} \hat{c}_{k}^{\dagger} \hat{d}_{m}+V_{m k} \hat{d}_{m}^{\dagger} \hat{c}_{k}\right),
$$

and $\hat{H}_{K}(K=L, R)$ represents the Hamiltonian of the contacts

$$
\hat{H}_{K}=\sum_{k \in K} \varepsilon_{k} \hat{c}_{k}^{\dagger} \hat{c}_{k}
$$

Here $\hat{c}_{k}^{\dagger}\left(\hat{c}_{k}\right)$ and $\hat{d}_{m}^{\dagger}\left(\hat{d}_{m}\right)$ are the creation (annihilation) operators for an electron in a state $k$ of the contact $K$ and in state $m$ of the molecule, respectively.

We introduce the many-body states of isolated molecule $|N, i\rangle$ with $N$, the number of electrons on the molecule, and $i$, the set of all the other quantum numbers characterizing a particular state of the molecule in the charging block $N$. These states are assumed to be orthonormal

$$
\left\langle N, i \mid N^{\prime}, i^{\prime}\right\rangle=\delta_{N, N^{\prime}} \delta_{i, i^{\prime}} .
$$

We note that nonorthogonal basis have also been considered in the literature. ${ }^{33}$ We use orthonormal basis for notational convenience. Molecular transitions (in our case due to coupling to the contacts) are naturally described in the language of Hubbard operators

$$
\hat{X}_{\left(N, i ; N^{\prime}, i^{\prime}\right)}=|N, i\rangle\left\langle N^{\prime}, i^{\prime}\right| .
$$

An important type of transition for our purpose correspond to the oxidation/reduction of the molecule by one electron. These transitions occur between neighboring charge blocks and will be labeled by

$$
\mathcal{M} \equiv(N, i, N+1, j) \quad \overline{\mathcal{M}} \equiv(N+1, j, N, i) .
$$

The molecular Hamiltonian can be written in terms of Hubbard operators as

$$
\hat{H}_{M}=\sum_{N, i, j}|N, i\rangle H_{i j}^{(N)}\langle N, j| \equiv \sum_{N, i, j} H_{i j}^{(N)} \hat{X}_{(N, i, N, j)} .
$$

If the many-body states are chosen to be the eigenstates of the molecular Hamiltonian, $H_{i j}^{(N)}=E_{i}^{(N)} \delta_{i, j}\left(E_{i}^{(N)}\right.$ is the energy of the molecular eigenstate $|N, i\rangle)$. The molecule-contacts coupling [Eq. (2)] represented in the language of many-body states reads

$$
\hat{V}=\sum_{k, \mathcal{M}}\left(V_{k \mathcal{M}} \hat{c}_{k}^{\dagger} \hat{X}_{\mathcal{M}}+V_{\overline{\mathcal{M} k}} \hat{X}_{\overline{\mathcal{M}}} \hat{c}_{k}\right),
$$

where

$$
V_{k \mathcal{M}} \equiv \sum_{m \in M} V_{k m}\left\langle N, i\left|\hat{d}_{m}\right| N+1, j\right\rangle
$$

and $V_{\overline{\mathcal{M} k}} \equiv V_{k \mathcal{M}}^{*}$. Note that $\hat{X}_{\overline{\mathcal{M}}}=\hat{X}_{\mathcal{M}}^{\dagger}$

As in Refs. 22 and 24, we introduce the many-body (Hubbard) GF on the Keldysh contour

$$
G_{(a, b),(c, d)}\left(\tau, \tau^{\prime}\right) \equiv-i\left\langle T_{c} \hat{X}_{a b}(\tau) \hat{X}_{c d}^{\dagger}\left(\tau^{\prime}\right)\right\rangle,
$$

where $a, b, c, d$ are many-body states of the isolated molecule, $T_{c}$ is the contour ordering operator, and $\tau, \tau^{\prime}$ are contour variables. The Hubbard operators evolve in time according to $\hat{X}_{a b}(t)=e^{i \hat{H} t} \hat{X}_{a b} e^{-i \hat{H} t}$, where $|a\rangle \equiv\left|N_{a}, s_{a}\right\rangle$ and $|b\rangle$ $\equiv\left|N_{b}, s_{b}\right\rangle$ are the many-body states defined in Eq. (4). The expectation value is defined as $\langle\cdot\rangle=\operatorname{Tr}\left\{\cdot \rho_{0}\right\}$ with the initial density matrix taken as usual at the infinite past.

Since we are interested in QME-like result we start with equation of motion for an object local in time. A useful choice (see below) is expectation value of the Hubbard operator, which obeys the Heisenberg equation of motion

$$
\frac{d\left\langle\hat{X}_{a b}(t)\right\rangle}{d t}=i\left\langle\left[\hat{H}, \hat{X}_{a b}(t)\right]\right\rangle .
$$

Evaluating the commutator in the right side of Eq. (11) yields (details are given in Appendix A) correlation functions of the form $\left\langle\hat{X}_{(\ldots)}^{\dagger}(t) \hat{c}_{k}(t)\right\rangle$ and $\left\langle\hat{c}_{k}^{\dagger}(t) \hat{X}_{(\ldots)}(t)\right\rangle$. As usual, ${ }^{34,35}$ these correlation functions can be treated as lesser projections of the GFs 


$$
\begin{aligned}
& G_{c X}\left(\tau, \tau^{\prime}\right)=-i\left\langle T_{c} \hat{c}_{k}(\tau) \hat{X}_{(\ldots)}^{\dagger}\left(\tau^{\prime}\right)\right\rangle, \\
& G_{X c}\left(\tau, \tau^{\prime}\right)=-i\left\langle T_{c} \hat{X}_{(\ldots)}(\tau) \hat{c}_{k}^{\dagger}\left(\tau^{\prime}\right)\right\rangle
\end{aligned}
$$

taken at equal time. The following procedure is similar to the one used by Meir and Wingreen ${ }^{32,34}$ to obtain a general expression for the electric current, and to that utilized by one of the authors to derive a general expression for the thermal current within NEGF. ${ }^{36}$ The GFs [Eqs. (12) and (13)] can be obtained by applying Langreth projection rules ${ }^{37}$ to on-thecontour equations of motion

$$
\begin{aligned}
G_{C X}\left(\tau, \tau^{\prime}\right) & =\sum_{\mathcal{M}} \int_{c} d \tau_{1} g_{k}\left(\tau, \tau_{1}\right) V_{k \mathcal{M}} G_{\mathcal{M}, \ldots}\left(\tau_{1}, \tau^{\prime}\right), \\
G_{X C}\left(\tau, \tau^{\prime}\right) & =\sum_{\mathcal{M}} \int_{c} d \tau_{1} G_{\ldots, \mathcal{M}}\left(\tau, \tau_{1}\right) V_{\mathcal{M} k} g_{k}\left(\tau_{1}, \tau^{\prime}\right) .
\end{aligned}
$$

Here $V_{k \mathcal{M}}\left(V_{\mathcal{M} k}\right)$ is defined in Eq. (9), $G_{\mathcal{M}, \ldots}\left(G_{\ldots, \mathcal{M}}\right)$ in Eq. (10), and

$$
g_{k}\left(\tau, \tau^{\prime}\right) \equiv-i\left\langle T_{c} \hat{c}_{k}(\tau) \hat{c}_{k}^{\dagger}\left(\tau^{\prime}\right)\right\rangle
$$

is the GF of the free electrons in the contacts. Using lesser projections taken at equal times of Eqs. (14) and (15) in Eq. (11) leads to (see Appendix A for details)

$$
\begin{aligned}
\frac{d\left\langle\hat{X}_{a b}(t)\right\rangle}{d t}= & \left.i \sum_{s}\left[H_{s s_{a}}^{\left(N_{a}\right)}\left\langle\hat{X}_{\left(N_{a}, s ; N_{b}, s_{b}\right.}(t)\right\rangle-H_{s_{b} s}^{\left(N_{b}\right)}\left\langle\hat{X}_{N_{a}, s ; a} ; N_{b}, s\right)\right\rangle\right] \\
& +\sum_{\mathcal{M}, s} \int_{-\infty}^{t} d t_{1}\left\{G_{\left(N_{a}, s_{a} ; N_{b}+1, s\right), \mathcal{M}}^{<}\left(t, t_{1}\right) \Sigma_{\mathcal{M},\left(N_{b}, s_{b} ; N_{b}+1, s\right)}^{>}\left(t_{1}-t\right)+\Sigma_{\left(N_{a}, s_{a} ; N_{a}+1, s\right), \mathcal{M}}^{>}\left(t-t_{1}\right) G_{\mathcal{M},\left(N_{b}, s_{b} ; N_{a}+1, s\right)}^{<}\left(t_{1}, t\right)\right. \\
& -G_{\left(N_{a}, s_{a} ; N_{b}+1, s\right), \mathcal{M}}^{>}\left(t, t_{1}\right) \Sigma_{\mathcal{M},\left(N_{b}, s_{b} ; N_{b}+1, s\right)}^{<}\left(t_{1}-t\right)-\Sigma_{\left(N_{a}, s_{a} ; N_{a}+1, s\right), \mathcal{M}}^{<}\left(t-t_{1}\right) G_{\mathcal{M},\left(N_{b}, s_{b} ; N_{a}+1, s\right)}^{>}\left(t_{1}, t\right) \\
& -(-1)^{N_{a}-N_{b}} \times\left[G_{\left(N_{a}-1, s ; N_{b}, s_{b}\right), \mathcal{M}}^{<}\left(t, t_{1}\right) \Sigma_{\mathcal{M},\left(N_{a}-1, s ; N_{a}, s_{a}\right)}^{>}\left(t_{1}-t\right)+\Sigma_{\left(N_{b}-1, s ; N_{b}, s_{b}\right), \mathcal{M}}^{>}\left(t-t_{1}\right) G_{\mathcal{M},\left(N_{b}, s_{b} ; N_{a}+1, s\right)}^{<}\left(t_{1}, t\right)\right. \\
& \left.\left.-G_{\left(N_{a}-1, s ; N_{b}, s_{b}\right), \mathcal{M}}^{>}\left(t, t_{1}\right) \Sigma_{\mathcal{M},\left(N_{a}-1, s ; N_{a}, s_{a}\right)}^{<}\left(t_{1}-t\right)-\Sigma_{\left(N_{b}-1, s ; N_{b}, s_{b}\right), \mathcal{M}}^{<}\left(t-t_{1}\right) G_{\mathcal{M},\left(N_{b}-1, s ; N_{a}, s_{a}\right)}^{>}\left(t_{1}, t\right)\right]\right\} .
\end{aligned}
$$

Here $\left.\Sigma_{\mathcal{M}_{1}, \mathcal{M}_{2}}^{>}<t\right)$ are the greater and lesser molecular selfenergies due to the coupling to the contacts

$$
\begin{gathered}
\sum_{\mathcal{M}_{1}, \mathcal{M}_{2}}^{>><}(t)=\sum_{K=L, R} \sum_{\mathcal{M}_{1}, \mathcal{M}_{2}}^{(K)>}(t), \\
\sum_{\mathcal{M}_{1}, \mathcal{M}_{2}}^{(K)><}(t)=\sum_{k \in K} V_{\mathcal{M}_{1}, k} g_{k}^{>,<}(t) V_{k, \mathcal{M}_{2}}
\end{gathered}
$$

with $G_{\mathcal{M}_{1}, \mathcal{M}_{2}}^{>,<}\left(t_{1}, t_{2}\right)$, the greater and lesser projections of Eq. (10)

$$
\begin{aligned}
& G_{(a ; b),(c ; d)}^{>}\left(t_{1}, t_{2}\right)=-i\left\langle\hat{X}_{a b}\left(t_{1}\right) \hat{X}_{c d}^{\dagger}\left(t_{2}\right)\right\rangle, \\
& G_{(a ; b),(c ; d)}^{<}\left(t_{1}, t_{2}\right)= \pm i\left\langle\hat{X}_{c d}^{\dagger}\left(t_{2}\right) \hat{X}_{a b}\left(t_{1}\right)\right\rangle .
\end{aligned}
$$

and $g_{k}^{>,<}(t)$, the greater and lesser projections of Eq. (16)

$$
\begin{gathered}
g_{k}^{>}(t)=-i\left[1-n_{k}\right] e^{-i \varepsilon_{k} t}, \\
g_{k}^{<}(t)=i n_{k} e^{-i \varepsilon_{k} t} .
\end{gathered}
$$

Note that in Eq. (21), "+" occurs when both $(a ; b)$ and $(c ; d)$ are transitions of Fermi type, and "-" otherwise. For future reference, we also define a damping matrix in Liouville space

$$
\Gamma_{\mathcal{M}_{1}, \mathcal{M}_{2}}^{(K)} \equiv i\left[\Sigma_{\mathcal{M}_{1}, \mathcal{M}_{2}}^{>(K)}-\Sigma_{\mathcal{M}_{1}, \mathcal{M}_{2}}^{<(K)}\right]
$$

The expression for the current, defined as $I_{K}(t) \equiv$ $-e \frac{d}{d t}\left\langle\hat{N}_{K}(t)\right\rangle$, where $\hat{N}_{K} \equiv \Sigma_{k \in K} \hat{c}_{k}^{\dagger} \hat{c}_{k}$, can be derived in a similar way [see Eq. 10 of Ref. 24]

$$
\begin{aligned}
I_{K}(t)= & \frac{e}{\hbar} \sum_{\mathcal{M}, \mathcal{M}^{\prime}} \int_{-\infty}^{t} d t^{\prime}\left\{\Sigma_{\mathcal{M}, \mathcal{M}^{\prime}}^{<}\left(t-t^{\prime}\right) G_{\mathcal{M}^{\prime}, \mathcal{M}}^{>}\left(t^{\prime}, t\right)\right. \\
& +G_{\mathcal{M}, \mathcal{M}^{\prime}}^{>}\left(t, t^{\prime}\right) \Sigma_{\mathcal{M}^{\prime}, \mathcal{M}}^{<}\left(t^{\prime}-t\right) \\
& -\Sigma_{\mathcal{M}, \mathcal{M}^{\prime}}^{>}\left(t-t^{\prime}\right) G_{\mathcal{M}^{\prime}, \mathcal{M}}^{<}\left(t^{\prime}, t\right) \\
& \left.-G_{\mathcal{M}, \mathcal{M}^{\prime}}^{<}\left(t, t^{\prime}\right) \Sigma_{\mathcal{M}^{\prime}, \mathcal{M}}^{>}\left(t^{\prime}-t\right)\right\} .
\end{aligned}
$$

Equations (17) and (25) are exact, however they are not closed in terms of $\left\langle\hat{X}_{a b}(t)\right\rangle$ since their right sides are expressed in terms of GFs. Our goal now is to introduce an 
approximate scheme to close Eq. (17) and thus find a connection with the QME.

\section{GENERALIZED QME}

Before introducing the ansatz that closes Eq. (17), wenote the connection between the equations of motion for $\hat{X}_{a b}(t)$ and density matrix element $\rho_{b a}(t)$. Indeed,

$$
\begin{aligned}
\rho_{b a}(t) & =\left\langle\left\langle\hat{X}_{b a}\left|e^{-i \mathcal{L} t}\right| \hat{\rho}_{0}\right\rangle\right\rangle=\left\langle\left\langle\hat{X}_{b a} \mid e^{-i \mathcal{L} t} \hat{\rho}_{0}\right\rangle\right\rangle=\left\langle\left\langle e^{i \mathcal{L} \dagger t} \hat{X}_{b a} \mid \hat{\rho}_{0}\right\rangle\right\rangle \\
& =\left\langle\hat{X}_{a b}(t)\right\rangle,
\end{aligned}
$$

where $\mathcal{L}$ is the total Liouvillian and $\langle\langle A \mid B\rangle\rangle \equiv \operatorname{Tr}\left[\hat{A}^{\dagger} \hat{B}\right]$ is the scalar product in Liouville space. Hence, closing Eq. (17) in terms of $\hat{X}_{a b}$ will result in a QME.

Correlation function (20) can be exactly written in Liouville space as

$$
\begin{aligned}
\left\langle\hat{X}_{a b}\left(t_{1}\right) \hat{X}_{c d}^{\dagger}\left(t_{2}\right)\right\rangle= & \theta\left(t_{1}-t_{2}\right)\left\langle\left\langle\hat{X}_{b a} \hat{I}_{K}\left|e^{-i \mathcal{L}\left(t_{1}-t_{2}\right)}\right| \hat{X}_{d c} \hat{\rho}\left(t_{2}\right)\right\rangle\right\rangle \\
& +\theta\left(t_{2}-t_{1}\right)\left\langle\left\langle\hat{X}_{c d} \hat{I}_{K}\left|e^{-i \mathcal{L}\left(t_{2}-t_{1}\right)}\right| \hat{\rho}\left(t_{1}\right) \hat{X}_{a b}\right\rangle\right\rangle .
\end{aligned}
$$

We now introduce the projector superoperator

$$
\left.\mathcal{P}=\sum_{e f}\left|\hat{X}_{e f} \hat{\rho}_{K}^{e q}\right\rangle\right\rangle\left\langle\left\langle\hat{X}_{e f} \hat{I}_{K}\right|,\right.
$$

which disregards nonequilibrium features in the leads and decouples the system and the bath dynamics. We propose, as an ansatz, to replace Eq. (27) by

$$
\begin{aligned}
\left\langle\hat{X}_{a b}\left(t_{1}\right) \hat{X}_{c d}^{\dagger}\left(t_{2}\right)\right\rangle \approx & \theta\left(t_{1}-t_{2}\right)\left\langle\left\langle\hat{X}_{b a} \hat{I}_{K}\left|e^{-i \mathcal{L}\left(t_{1}-t_{2}\right)} \mathcal{P}\right| \hat{X}_{d c} \hat{\rho}\left(t_{2}\right)\right\rangle\right\rangle \\
& +\theta\left(t_{2}-t_{1}\right)\left\langle\left\langle\hat{X}_{c d} \hat{I}_{K}\left|e^{-i \mathcal{L}\left(t_{2}-t_{1}\right)} \mathcal{P}\right| \hat{\rho}\left(t_{1}\right) \hat{X}_{a b}\right\rangle\right\rangle .
\end{aligned}
$$

Next, we introduce the retarded and advanced GFs in Liouville space (see Appendix B)

$$
\begin{aligned}
\mathcal{G}_{i j, m n}^{r}(t) & \equiv-i \theta(t)\left\langle\left\langle\hat{X}_{j i} \hat{I}_{K}\left|e^{-i \mathcal{L} t}\right| \hat{X}_{n m} \hat{\rho}_{K}^{e q}\right\rangle\right\rangle \\
& =-i \theta(t)\left\langle\left\langle\hat{X}_{j i}\left|\mathcal{U}_{\mathrm{eff}}(t)\right| \hat{X}_{n m}\right\rangle\right\rangle, \\
\mathcal{G}_{i j, m n}^{a}(t) & \equiv i \theta(-t)\left\langle\left\langle\hat{X}_{m n} \hat{I}_{K}\left|e^{i \mathcal{L} t}\right| \hat{X}_{i j} \hat{\rho}_{K}^{e q}\right\rangle\right\rangle \\
& =i \theta(-t)\left\langle\left\langle\hat{X}_{j i}\left|\mathcal{U}_{\mathrm{eff}}^{\dagger}(-t)\right| \hat{X}_{n m}\right\rangle\right\rangle,
\end{aligned}
$$

where the effective propagator in the molecule space reads

$$
\mathcal{U}_{\mathrm{eff}}(t) \equiv\left\langle\left\langle\cdot \hat{I}_{K}\left|e^{-i \mathcal{L} t}\right| \cdot \hat{\rho}_{K}^{\mathrm{eq}}\right\rangle\right\rangle=\operatorname{Tr}_{K}\left\{e^{-i \mathcal{L} t} \hat{\rho}_{K}^{\mathrm{eq}}\right\} .
$$

Using Eqs. (30) and (31), we can rewrite Eq. (29) as

$$
\begin{aligned}
\left\langle\hat{X}_{a b}\left(t_{1}\right) \hat{X}_{c d}^{\dagger}\left(t_{2}\right)\right\rangle= & i \sum_{e, f}\left[\mathcal{G}_{a b, f e}^{r}\left(t_{1}-t_{2}\right)\left\langle\hat{X}_{f e}\left(t_{2}\right) \hat{X}_{c d}^{\dagger}\left(t_{2}\right)\right\rangle\right. \\
& \left.-\left\langle\hat{X}_{a b}\left(t_{1}\right) \hat{X}_{e f}^{\dagger}\left(t_{1}\right)\right\rangle \mathcal{G}_{e f, c d}^{a}\left(t_{1}-t_{2}\right)\right] \\
\equiv & i \sum_{m}\left[\mathcal{G}_{a b, m d}^{r}\left(t_{1}-t_{2}\right)\left\langle\hat{X}_{m c}\left(t_{2}\right)\right\rangle\right. \\
& \left.-\left\langle\hat{X}_{a m}\left(t_{1}\right)\right\rangle \mathcal{G}_{m b, c d}^{a}\left(t_{1}-t_{2}\right)\right]
\end{aligned}
$$

where second equality follows from the orthonormality condition (4).

Similar consideration for correlation function (21) leads to

$$
\begin{aligned}
\left\langle\hat{X}_{c d}^{\dagger}\left(t_{2}\right) \hat{X}_{a b}\left(t_{1}\right)\right\rangle= & i \sum_{e, f}\left[\mathcal{G}_{a b, f e}^{r}\left(t_{1}-t_{2}\right)\left\langle\hat{X}_{c d}^{\dagger}\left(t_{2}\right) \hat{X}_{f e}\left(t_{2}\right)\right\rangle\right. \\
& \left.-\left\langle\hat{X}_{e f}^{\dagger}\left(t_{1}\right) \hat{X}_{a b}\left(t_{1}\right)\right\rangle \mathcal{G}_{e f, c d}^{a}\left(t_{1}-t_{2}\right)\right] \\
\equiv & i \sum_{m}\left[\mathcal{G}_{a b, c m}^{r}\left(t_{1}-t_{2}\right)\left\langle\hat{X}_{d m}\left(t_{2}\right)\right\rangle\right. \\
& \left.-\left\langle\hat{X}_{m b}\left(t_{1}\right)\right\rangle \mathcal{G}_{a m, c d}^{a}\left(t_{1}-t_{2}\right)\right]
\end{aligned}
$$

It is interesting to note that Eqs. (33) and (34) can be considered as the Liouville space analog of the generalized Kadanoff-Baym ansatz. ${ }^{32}$

Using Eqs. (33) and (34) in Eq. (17) we close the latter in terms of the density matrix $\rho_{b a}(t) \equiv\left\langle\hat{X}_{a b}(t)\right\rangle$

$$
\begin{aligned}
& \frac{d \rho_{12}(t)}{d t}=-i \sum_{3,4}\left\{\delta_{N_{1}, N_{3}} \delta_{N_{2}, N_{4}} \sum_{s}\left[\delta_{i_{2}, i_{4}} H_{i_{1}, i_{3}}^{\left(N_{1}\right)}-\delta_{i_{1}, i_{3}} H_{i_{4}, i_{2}}^{\left(N_{2}\right)}\right] \rho_{34}(t)-i \sum_{s_{1}, s_{2}} \int_{-\infty}^{+\infty} d t_{1}\right. \\
& \times\left[\mathcal{G}_{\left(2 ; N_{1}+1, s_{1}\right)\left(4 ; N_{3}+1, s_{2}\right)}^{r}\left(t-t_{1}\right) \Sigma_{\left(3 ; N_{3}+1, s_{2}\right)\left(1 ; N_{1}+1, s_{1}\right)}^{<}\left(t_{1}-t\right)-\Sigma_{\left(2 ; N_{2}+1, s_{1}\right)\left(4 ; N_{4}+1, s_{2}\right)}^{<}\left(t-t_{1}\right) \mathcal{G}_{\left(3 ; N_{4}+1, s_{2}\right)\left(1 ; N_{2}+1, s_{1}\right)}^{a}\left(t_{1}-1\right)\right. \\
& -\mathcal{G}_{\left(N_{2}-1, s_{1} ; 1\right)\left(N_{4}-1, s_{2} ; 3\right)}^{r}\left(t-t_{1}\right) \Sigma_{\left(N_{4}-1, s_{2} ; 4\right)\left(N_{2}-1, s_{1} ; 2\right)}^{>}\left(t_{1}-t\right)+\Sigma_{\left(N_{1}-1, s_{1} ; 1\right)\left(N_{3}-1, s_{2} ; 3\right)}^{>}\left(t-t_{1}\right) \mathcal{G}_{\left(N_{3}-1, s_{2} ; 4\right)\left(N_{1}-1, s_{1}, 2\right)}^{a}\left(t_{1}-t\right) \\
& \text { - }(-1)^{N_{1}-N_{2}}\left(\mathcal{G}_{\left(N_{2}-1, s_{1} ; 1\right)\left(4 ; N_{3}+1, s_{2}\right)}^{r}\left(t-t_{1}\right) \sum_{\left(3 ; N_{3}+1, s_{2}\right)\left(N_{2}-1, s_{1} ; 2\right)}^{<}\left(t_{1}-t\right)-\Sigma_{\left(N_{1}-1, s_{1} ; 1\right)\left(4 ; N_{4}+1, s_{2}\right)}^{<}\left(t-t_{1}\right) \mathcal{G}_{\left(3 ; N_{4}+1, s_{2}\right)\left(N_{1}-1, s_{1} ; 2\right)}^{a}\left(t_{1}-1\right)\right. \\
& \left.\left.\left.-\mathcal{G}_{\left(2 ; N_{1}+1, s_{1}\right)\left(N_{4}-1, s_{2} ; 3\right)}^{r}\left(t-t_{1}\right) \Sigma_{\left(N_{4}-1, s_{2} ; 4\right)\left(1 ; N_{1}+1, s_{1}\right)}^{>}\left(t_{1}-t\right)+\Sigma_{\left(2 ; N_{2}+1, s_{1}\right)\left(N_{3}-1, s_{2} ; 3\right)}^{>}\left(t-t_{1}\right) \mathcal{G}_{\left(N_{3}-1, s_{2} ; 4\right)\left(1 ; N_{s}+1, s_{1}\right)}^{a}\left(t_{1}-t\right)\right)\right] \rho_{34}\left(t_{1}\right)\right\} \text {. }
\end{aligned}
$$


This is a generalized non-Markovian QME. Note that prefactor $(-1)^{N_{1}-N_{2}}$ coming from coherences between different charge blocks is usually missed in the standard QME derivations.

To make Eq. (35) more tractable, we can assume a Markovian generator $\mathcal{L}_{\text {eff }}$ (e.g., Markovian Redfield generator $^{38-40}$ ) for the retarded and advanced GFs [Eqs. (30) and (31)]

$$
\mathcal{U}_{\mathrm{eff}}(t) \approx e^{-i \mathcal{L}_{\mathrm{eff}} t}
$$

so that

$$
\begin{aligned}
& \mathcal{G}_{i j, m n}^{r}(t) \equiv-i \theta(t)\left\langle\left\langle\hat{X}_{j i}\left|e^{-i \mathcal{L}_{\text {eff }} t}\right| \hat{X}_{n m}\right\rangle\right\rangle, \\
& \mathcal{G}_{i j, m n}^{a}(t) \equiv i \theta(-t)\left\langle\left\langle\hat{X}_{j i} \mid e^{-i \mathcal{L}_{\text {eff }}^{\dagger} \mid} \hat{X}_{n m}\right\rangle\right\rangle .
\end{aligned}
$$

The ansatz (29) together with Eqs. (37) and (38) is equivalent to use of the regression formula on the Hubbard GFs. This procedure is often employed to calculate multipoint correlation functions using effective Markovian propagators. ${ }^{38,39,41}$

Equation (35) is the main result of this paper. It generalizes the standard QME by effectively dressing the evolution operator. The only approximation used when deriving Eq. (35) from the exact Eq. (17) is the approximate treatment of the time correlation using the ansatz (29). This loss of information on the time correlation is the main difference between the DM (local in time) and the GF (nonlocal in time) techniques. Thus, Eq. (35) is an approximate nonperturbative result. Note that if $\mathcal{L}_{\text {eff }}$ is taken to be the Redfield generator, the equation becomes effectively of second order in the system-bath coupling. Equation (35) also allows to treat level broadening which is lost in the standard QME. Technically, within GF techniques, the level broadening is introduced through the imaginary part of the retarded (advanced) selfenergy which enters the expression for the retarded (advanced) GF. ${ }^{32}$ Neglecting these self-energies corresponds to the substitution of the full (dressed) GFs in Eq. (35) by the zero-order (free or undressed evolution) GFs. In our approach this is accomplished by introducing the free molecular evolution $\mathcal{L}_{M}=\left[\hat{H}_{M} ;\right]$ instead of the effective one $\mathcal{L}_{\text {eff }}$ in Eqs. (37) and (38). This transforms Eq. (35) to the standard non-Markovian QME. ${ }^{21}$ The difference between standard and generalized versions of QME is therefore similar to the dressing of the diagrams in GF diagrammatic technique.

Below we use the generator of the Markovian Redfield equation to get $\mathcal{L}_{\text {eff }}$ (see Appendix C). Its spectral decomposition reads

$$
\left.\mathcal{L}_{\text {eff }}=\sum_{\gamma}\left|R_{\gamma}\right\rangle\right\rangle \lambda_{\gamma}\left\langle\left\langle L_{\gamma}\right|\right.
$$

The eigenvalues $\lambda_{\gamma}$ and the left $\left.\left|L_{\gamma}\right\rangle\right\rangle$ and right $\left.\left|R_{\gamma}\right\rangle\right\rangle$ eigenvectors provide a numerically tractable scheme to deal with generalized QME [Eq. (35)] by utilizing

$$
\begin{aligned}
& \mathcal{G}_{i j, m n}^{r}(t)=-i \theta(t) \sum_{\gamma}\left\langle\left\langle j i \mid R_{\gamma}\right\rangle\right\rangle e^{-i \lambda \gamma^{t}}\left\langle\left\langle L_{\gamma} \mid n m\right\rangle\right\rangle, \\
& \mathcal{G}_{i j, m n}^{a}(t)=i \theta(-t) \sum_{\gamma}\left\langle\left\langle j i \mid L_{\gamma}\right\rangle\right\rangle e^{-i \lambda^{*} t}\left\langle\left\langle R_{\gamma} \mid n m\right\rangle\right\rangle .
\end{aligned}
$$

The steady state of Eq. (35) is given by the right eigenvector with zero eigenvalue of the Liouvillian corresponding to the Markov limit of Eq. (35).

Similarly, an approximate expression for current in terms of $\left\langle\hat{X}_{(\ldots)}\right\rangle$ can be obtained using Eqs. (33) and (34) in Eq. (25)

$$
\begin{aligned}
I_{K}(t)= & \frac{e}{\hbar} \sum_{\mathcal{M}_{1}, \mathcal{M}_{2}} \sum_{e} \int_{-\infty}^{+\infty} d t_{1} 2 \operatorname{Re}\left[\mathcal{G}_{\left(N_{1}, i_{1} ; N_{1}+1, j_{1}\right),\left(N_{2}, i_{2} ; e\right)}^{r}\left(t-t_{1}\right) \Sigma_{\left(N_{2}, i_{2} ; N_{2}+1, j_{2}\right),\left(N_{1}, i_{1} ; N_{1}+1, j_{1}\right)}^{>}\left(t_{1}-t\right)\left\langle\hat{X}_{\left(N_{2}+1, j_{2} ; e\right)}\left(t_{1}\right)\right\rangle\right. \\
& \left.+\mathcal{G}_{\left(N_{1}, i_{1} ; N_{1}+1, j_{1}\right),\left(e ; N_{2}+1, j_{2}\right)}^{r}\left(t-t_{1}\right) \Sigma_{\left(N_{2}, i_{2} ; N_{2}+1, j_{2}\right),\left(N_{1}, i_{1} ; N_{1}+1, j_{1}\right)}^{<}\left(t_{1}-t\right)\left\langle\hat{X}_{\left(e ; N_{2}, i_{2}\right)}\left(t_{1}\right)\right\rangle\right] .
\end{aligned}
$$

The theory presented in this section is suited to treat transport in the Coulomb blockade regime, i.e., in the case of relatively weak coupling between the system (molecule) and the leads. Since numerically tractable scheme accounts for system-bath coupling within effective second order, the Kondo regime is beyond its capabilities. The presented approach is more general than the standard QME considerations because it takes into account the broadening of the isolated molecule levels. While QME-type considerations are popular due to their ability to deal with transport in the many-body molecular states language (all the on-themolecule correlations are taken into account by construction), they often fail to go beyond resonant hopping mechanism. GF techniques treat both hopping (resonant) and superexchange (off-resonant) cases in a unified manner by taking into account molecular level broadening due to coupling to the leads (when relaxation mechanism is present in the system, the two mechanisms are two extremes, determined by relative position of electron tunneling energy relative to the closest resonance). However, they are usually formulated in the language of effective single-particle orbitals, which becomes inadequate in the case of near-resonant transport, when actual oxidation/reduction of the molecule occurs. The latter may lead to essential changes in the electronic structure of the system. It is this regime which is of utter importance for construction of molecular logic and memory devices. Attempts to combine the NEGF approach with a transport description in the language of molecular states have 
been done by one of the authors and others (see, e.g., Ref. 24 and references therein). The formulation is however quite complicated and numerically demanding. The present theory introduces a "lighter" approach by making a bridge between QME and GF techniques. It keeps the relative simplicity of QME and its ability to treat transport in the language of many-body states, while still keeping (albeit approximately) the NEGF capability to treat both hopping and superexchange in a unified manner.

Our approach has potentially a direct relation to molecular studies since it allows the incorporation of powerful quantum chemistry electronic structure methods (done for an isolated molecule with well-defined number of electrons on it) into transport calculations (where state of the molecule is a complicated mixture of many-body states and their coherences). As a lighter alternative of the scheme proposed in Ref. 24, it should be more easily applicable to realistic calculations. Note that standard QME calculations on realistic molecular junctions are available in the literature.

\section{RESULTS AND DISCUSSION}

In this section we illustrate our theory by applying it to simple models.

\section{A. Resonant level model}

As a first example we consider a simple resonant-level model. One has two charge blocks (occupied and unoccupied level) with one state in each of them: $|0\rangle$ and $|1\rangle$. The molecular Hamiltonian is

$$
\hat{H}_{M}=|1\rangle \varepsilon_{0}\langle 1| .
$$

The current Eq. (42) in this case becomes

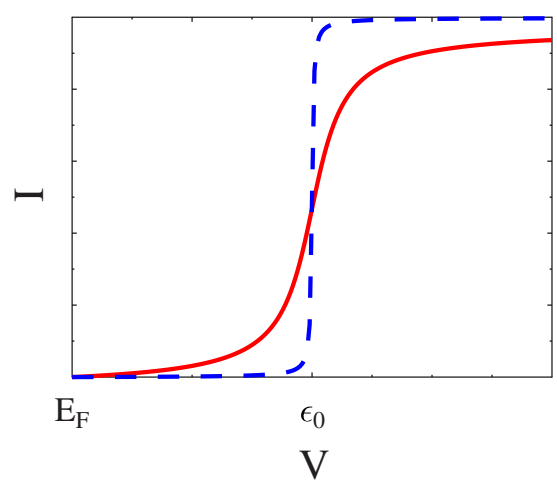

FIG. 1. (Color online) Current-voltage characteristic for resonant level model (43). The generalized QME (solid line, red) is compared to the standard QME (dashed line, blue) result. Note that the generalized QME result is exact for this model. See text for parameters.

$$
\begin{aligned}
I_{K}(t)= & \frac{i e}{\hbar} \int_{-\infty}^{t} d t_{1}\left\{\left[\mathcal{G}_{01,01}^{r}\left(t-t_{1}\right) \Sigma_{K}^{>}\left(t_{1}-t\right)\right.\right. \\
& \left.-\Sigma_{K}^{>}\left(t-t_{1}\right) \mathcal{G}_{01,01}^{a}\left(t_{1}-t\right)\right] \rho_{11}\left(t_{1}\right) \\
& +\left[\mathcal{G}_{01,01}^{r}\left(t-t_{1}\right) \Sigma_{K}^{<}\left(t_{1}-t\right)\right. \\
& \left.\left.-\Sigma_{K}^{<}\left(t-t_{1}\right) \mathcal{G}_{01,01}^{a}\left(t_{1}-t\right) \rho_{00}\left(t_{1}\right)\right]\right\},
\end{aligned}
$$

where

$$
\begin{aligned}
& \mathcal{G}_{01,01}^{r}(t)=-i \theta(t) e^{-i\left(\varepsilon_{0}-i \Gamma / 2\right) t} \equiv G^{r}(t), \\
& \mathcal{G}_{01,01}^{a}(t)=i \theta(-t) e^{-i\left(\varepsilon_{0}+i \Gamma / 2\right) t} \equiv G^{a}(t),
\end{aligned}
$$

and $\Gamma=\Sigma_{K=L, R} \Gamma_{01,01}^{K}$ with $\Gamma_{0,1 ; 0,1}^{K} \equiv 2 \pi \Sigma_{k \in K}\left|V_{k}\right|^{2} \delta\left(E-\varepsilon_{k}\right)$ defined in Eq. (24).

The generalized QME [Eq. (17)] for this model becomes

$$
\begin{aligned}
\frac{d \rho_{11}(t)}{d t}= & -\frac{d \rho_{00}(t)}{d t}=\int_{-\infty}^{+\infty} d t_{1}\left\{\left[\mathcal{G}_{01,01}^{r}\left(t-t_{1}\right) \Sigma^{>}\left(t_{1}-t\right)-\Sigma^{>}\left(t-t_{1}\right) \mathcal{G}_{01,01}^{a}\left(t_{1}-t\right)\right] \rho_{11}\left(t_{1}\right)\right. \\
& \left.+\left[\mathcal{G}_{01,01}^{r}\left(t-t_{1}\right) \Sigma^{<}\left(t_{1}-t\right)-\Sigma^{<}\left(t-t_{1}\right) \mathcal{G}_{01,01}^{a}\left(t_{1}-t\right)\right] \rho_{00}\left(t_{1}\right)\right\}
\end{aligned}
$$

At steady state, populations are independent of time. Taking the Fourier transform of GFs and SEs in Eq. (47) one gets

$$
\rho_{11}=1-\rho_{00}=n_{0},
$$

where the average occupation of the level is

$$
n_{0}=\int_{-\infty}^{+\infty} \frac{d E}{2 \pi} A(E)\left[\frac{\Gamma_{L}}{\Gamma} f_{L}(E)+\frac{\Gamma_{R}}{\Gamma} f_{R}(E)\right]
$$

and the spectral function is given by

$$
A(E)=\frac{\Gamma}{\left(E-\varepsilon_{0}\right)^{2}+(\Gamma / 2)^{2}} .
$$

$f_{K}(E)$ is the Fermi distribution in contact $K=L, R$. Using Eqs. (45), (46), and (48) in Eq. (44), we get the Landauer expression

$$
I_{K}=\frac{e}{\hbar} \int_{-\infty}^{+\infty} \frac{d E}{2 \pi} \frac{\Gamma_{L} \Gamma_{R}}{\Gamma} A(E)\left[f_{L}(E)-f_{R}(E)\right] .
$$

As announced, the generalized QME approach takes level broadening into account in a natural way contrary to the standard QME considerations. Note also that in this simple 


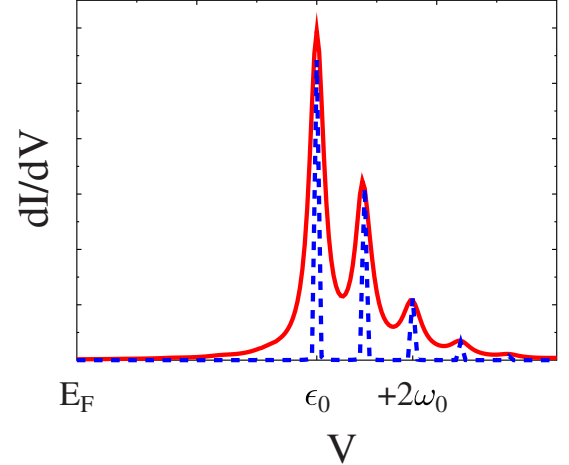

FIG. 2. (Color online) Conductance vs applied bias for the model of a resonant level coupled to a single vibration [Eq. (52)]. Generalized QME (solid line, red) is compared to standard QME (dashed line, blue) result. See text for parameters.

case our approximate scheme provides the exact result. ${ }^{42}$ It is not true in more complicated situations, and generally broadening is accounted for in an approximate way only.

Figure 1 compares results of calculation within our generalized QME (solid line) and standard QME (dashed line) approaches for current-voltage characteristic of single resonant-level $\varepsilon_{0}$ model. Generalized QME accounts for level broadening due to coupling to the contacts, while standard QME approach misses the broadening altogether. Parameters of the calculation are $\varepsilon_{0}=1, \Gamma_{L}=\Gamma_{R}=0.1, E_{F}=\mu_{R}$ $=0$, and $\mu_{L}=E_{F}+|e| V_{s d}$. Here and below we use arbitrary units.

\section{B. Resonant level coupled to vibration}

As a second example, we consider a resonant level $\varepsilon_{0}$ linearly coupled to a single vibration $\omega_{0}$

$$
\hat{H}_{M}=\varepsilon_{0} \hat{d}^{\dagger} \hat{d}+\omega_{0} \hat{a}^{\dagger} \hat{a}+M\left(\hat{a}+\hat{a}^{\dagger}\right) \hat{d}^{\dagger} \hat{d} .
$$

After the small polaron transformation, ${ }^{42}$ the coupling is removed and the shift operators $\hat{\mathcal{X}}=\exp \left[-M\left(\hat{a}^{\dagger}-\hat{a}\right) / \omega_{0}\right]$ are introduced in the molecule-contacts couplings

$$
\hat{V}=\sum_{k \in\{L, R\}}\left(V_{k}^{*} \hat{\mathcal{X}} \hat{c}_{k}^{\dagger} \hat{d}+V_{k} \hat{\mathcal{X}}^{\dagger} \hat{d}^{\dagger} \hat{c}_{k}\right)
$$

Going to the many-body states representation for electronic degrees of freedom along the lines presented in Eqs. (7)-(9), leads to

$$
\hat{H}=\bar{\varepsilon}_{0} \hat{X}_{0,1}+\omega_{0} \hat{a}^{\dagger} \hat{a}+\sum_{k \in\{L, R\}}\left(V_{k}^{*} \hat{\mathcal{X}} \hat{c}_{k}^{\dagger} \hat{X}_{0,1}+V_{k} \hat{\mathcal{X}}^{\dagger} \hat{X}_{0,1}^{\dagger} \hat{c}_{k}\right),
$$

where $\bar{\varepsilon}_{0}=\varepsilon_{0}-M^{2} / \omega_{0}$. As usual, assuming thermal distribution for the vibration, one gets the self-energies dressed by the Franck-Condon factors (for details see, e.g., Ref. 26).

Figure 2 shows conductance vs bias for the model of a single level $\varepsilon_{0}$ coupled to a vibration $\omega_{0}$. The generalized QME provides results similar to that of the NEGF consideration of Ref. 26 (see Fig. 4 there). The standard QME approach only predicts the positions of the peaks. The param-


FIG. 3. (Color online) Nondegenerate, $\varepsilon_{\uparrow} \neq \varepsilon_{\downarrow}$, quantum dot (QD) model. (a) Current-voltage characteristic compares generalized (solid line, red) and standard QME (dotted line, blue) approaches. Calculation is done for $V_{g}=-0.55$. (b) Conductance vs applied bias $V_{s d}$ and gate voltage $V_{g}$ calculated within generalized QME approach.

eters of the calculation are $\Gamma_{L}=\Gamma_{R}=0.05, \omega_{0}=0.2$, and $M$ $=0.2$. The other parameters are as in Fig. 1. In the simulations we used a small but finite broadening for the standard QME approach in order to avoid delta-function divergences



FIG. 4. (Color online) Conductance vs applied bias $V_{s d}$ and gate voltage $V_{g}$ for a degenerate quantum dot (QD) coupled to a vibration. The calculation is done using the generalized QME approach. The vibration frequency is $\omega_{0}=0.1$ and the strength of the electronvibration coupling is $M=0.1$. Other parameters are the same as in Fig. 3, except that $\varepsilon_{\uparrow}=\varepsilon_{\downarrow}=-0.5$ (degenerate case). 


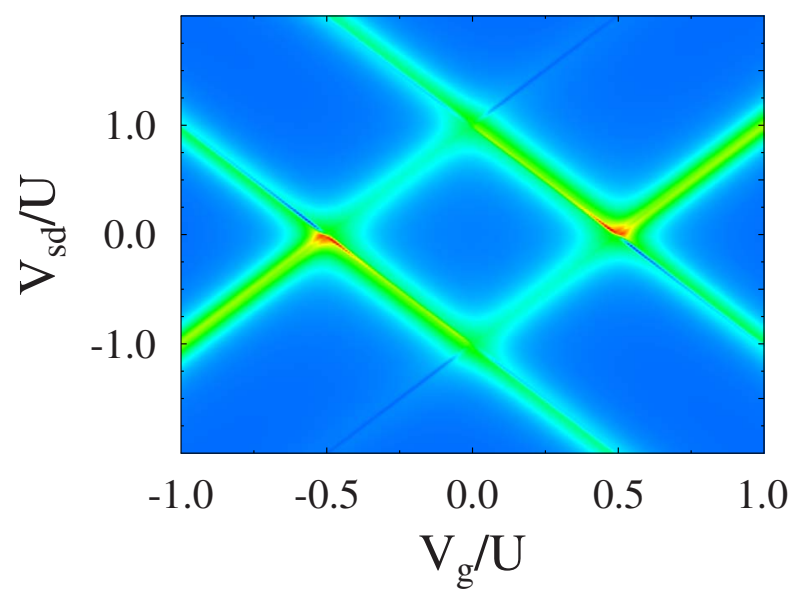

FIG. 5. (Color online) Conductance vs applied bias $V_{s d}$ and gate voltage $V_{g}$ for a degenerate QD asymmetrically coupled to the contacts. The calculation is performed using the generalized QME approach. Parameters used in the calculation are the same as in Fig. 3 except that $\varepsilon_{\uparrow}=\varepsilon_{\downarrow}=-0.5$ and $\Gamma_{L, \sigma}=0.01$ and $\Gamma_{R, \sigma}=0.1$.

in conductance. We also scaled the standard QME result in Fig. 1 for convenience.

\section{Quantum dot}

Next we consider a quantum dot. The many-body states are $|0\rangle,|\uparrow\rangle,|\downarrow\rangle$, and $|2\rangle$. They correspond, respectively, to an empty, singly (two-spin projections), and doubly occupied level. The molecular Hamiltonian is

$$
\hat{H}_{M}=\sum_{\sigma=\{\uparrow, \downarrow\}} \varepsilon_{\sigma} \hat{X}_{\sigma, \sigma}+\left(\varepsilon_{\uparrow}+\varepsilon_{\downarrow}+U\right) \hat{X}_{2,2} .
$$

A gate potential $V_{g}$ is used to shift position of the levels.

Figure 3 shows the current-voltage characteristic [Fig. 3(a)] and the conductance map [Fig. 3(b)] of a nondegenerate quantum dot. Parameters in the calculation are the level positions $\varepsilon_{\uparrow}=-0.4$ and $\varepsilon_{\downarrow}=-0.6$, the molecule-contacts coupling $\Gamma_{K, \sigma}=0.01(K=\{L, R\})$, the on-site repulsion $U=1$, and the Fermi level $E_{F}=0$. The electrochemical potentials in the contacts are $\mu_{L}=E_{F}+|e| V_{s d} / 2$ and $\mu_{R}=E_{F}-|e| V_{s d} / 2$. Figure 3(a) compares the generalized (solid line) and standard (dotted line) results. We verified that the results obtained within the generalized QME approach are similar to that obtained using the many-body GF technique of Ref. 24. Figure 3(b) shows the conductance map obtained using the generalized QME approach. For a discussion on the origin and intensity of the peaks, we refer to Ref. 43.

Figure 4 shows the conductance map for a model of a quantum dot coupled linearly to vibration $\omega_{0}$. The vibration is introduced as in Eqs. (52)-(54). The parameters of the calculation are the same as in Fig. 3, except that $\varepsilon_{\uparrow}=\varepsilon_{\downarrow}=$ -0.5 . The vibration frequency and coupling strength are $\omega_{0}$ $=0.1$ and $M=0.1$, respectively. In addition to the elastic peaks, the vibrational sidebands corresponding to resonant inelastic tunneling are reproduced as well.

Note that vibrations in both Figs. 2 and 4 are introduced, as is usually done in resonant inelastic transport consider-
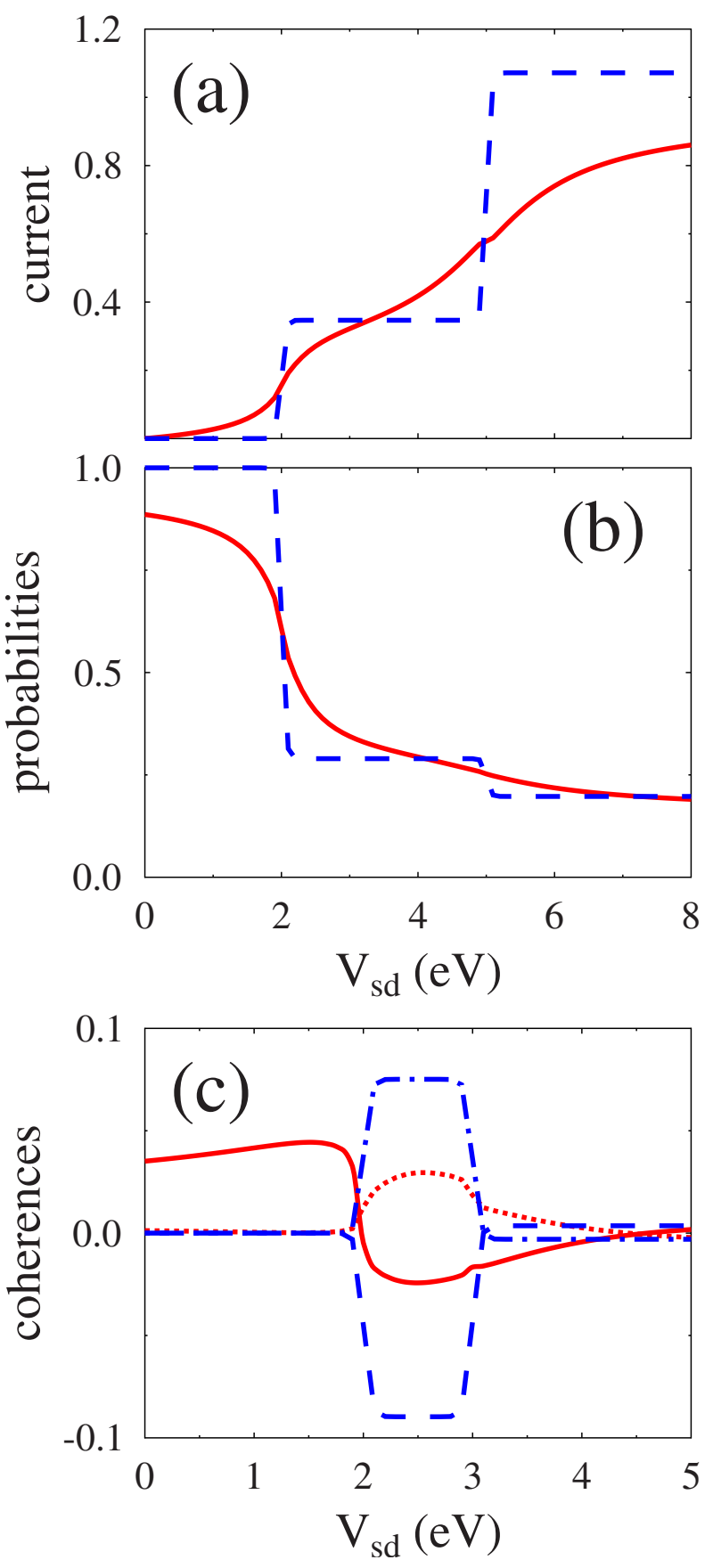

FIG. 6. (Color online) Two-level bridge model. Comparison between the prediction of the generalized QME of the present paper and the standard QME of Ref. 21. Shown are the current (a) and the probability for the system to be unoccupied (b) vs the applied biasgeneralized (solid line, red) and standard (dashed line, blue) QME considerations. (c) represents the real (solid, red and dashed, blue) and imaginary (dotted, red and dash-dotted, blue) parts of the coherences in the local eigenbasis vs the applied bias for the generalized (red) and the standard (blue) QME treatment, respectively. See text for parameters.

ations, with the help of the small polaron transformation. Therefore, the vibrational features in the electron transport stem from the Franck-Condon factors calculated under the 
assumption of an unperturbed thermal distribution of the vibrational populations. Actual vibrational states are not included in the current consideration. Some treatments go beyond this assumption by introducing a self-consistent procedure capable of treating approximately the influence of the electron flux on the vibrations and vice versa (see, e.g., Ref. 26). Still approximation of decoupling between electron and vibration degrees of freedom, leading to appearance of Franck-Condon factors, is employed. A way to incorporate the vibrational states into our many-body state picture, and to avoid the decoupling between the electron and vibration degrees of freedom, will be described elsewhere.

Figure 5 shows the conductance map for a degenerate quantum dot. $\varepsilon_{\uparrow}=\varepsilon_{\downarrow}=-0.5$, with asymmetric coupling to the contacts $\Gamma_{L, \sigma}=0.01$ and $\Gamma_{R, \sigma}=0.1$. This result reproduces the one presented in Fig. 4 of Ref. 18.

\section{Two-level bridge}

Finally, we consider a model of a two-level bridge

$$
\hat{H}_{M}=\sum_{m=1,2} \varepsilon_{m} \hat{d}_{m}^{\dagger} \hat{d}_{m},
$$

with couplings to the contacts given by Eq. (2). This model was previously considered in Ref. 21 using the standard QME approach. The authors studied the influence of coherences induced by the coupling to the contacts on the transport properties of the junction.

Figure 6 presents the comparison between the standard and generalized QME approaches. The parameters of the calculation are similar to those of Ref. 21: eigenenergies of the bridge are $\varepsilon_{1}=2 \mathrm{eV}$ and $\varepsilon_{2}=5 \mathrm{eV}$, strength of their coupling to contacts is $T_{1}^{L}=T_{2}^{L}=0.3 \mathrm{eV}, T_{1}^{R}=0.2 \mathrm{eV}$, and $T_{2}^{R}=0.4 \mathrm{eV}$. Here, $T_{m K} T_{K m^{\prime}} \equiv \sum_{k \in K} V_{m k} V_{k m^{\prime}} \delta\left(E-\varepsilon_{k}\right)$ are taken independent of the energy $E$ (wide-band approximation). For temperature, we take a physically reasonable value of $T$ $=0.03 \mathrm{eV}$.

Figures 6(a) and 6(b) show the current and one of the probabilities (probability of the system to be unoccupied) vs the applied bias. One sees that the broadening due to the coupling to the contacts is preserved in our scheme. Note also that the broadening presented in Ref. 21 was due to the artificially high values of temperature chosen. Figure 6(c) demonstrates the influence of the broadening on coherences (in local basis). Here, we bring the two eigenenergies closer to each other, $\varepsilon_{2}=3 \mathrm{eV}$, in order to make the coherences more pronounced. One sees that including the level broadening significantly changes the coherences.

\section{CONCLUSION}

The necessity for describing molecular transport in the language of many-body (isolated molecule) states has been recently realized to be essential for the description of resonant tunneling and for the study of optoelectronic devices, and several approaches were proposed. ${ }^{8-13,15,16,22-24}$ In this paper we introduced an approach alternative to the Hubbard operator GF scheme considered for inelastic transport in Ref. 24. This much simpler approach is formulated for the density matrix instead of the GF and provides a more easy way to calculate both time-dependent and steady-state transport in molecular junctions. Starting from GF-type consideration we introduce the Liouville space analog of the generalized Kadanoff-Baym ansatz, which allows us to derive a generalized QME. The latter incorporates an effective system propagation inside the generator instead of the free evolution. The procedure is similar in spirit to diagrams dressing in GF diagrammatic techniques. The capabilities of the scheme has been demonstrated on various model calculations. Application of this approach to optoelectronic response of molecular junctions is a goal for future research.

\section{ACKNOWLEDGMENTS}

M.E. is funded by the FNRS Belgium (chargé de recherche) and by the Government of Luxembourg (bourse de formation-recherche). M.G. gratefully acknowledges support from the UCSD Startup Fund and the UC Academic Senate research grant. This work was performed, in part, at the Center for Integrated Nanotechnologies, a (U.S.) Department of Energy, Office of Basic Energy Sciences user facility at Los Alamos National Laboratory under Contract No. DE-AC5206NA25396. M.G. thanks Tomáš Novotný for helpful discussion.

\section{APPENDIX A: DERIVATION OF EQ. (17)}

We start from Eq. (11), which, after evaluating the commutator, becomes

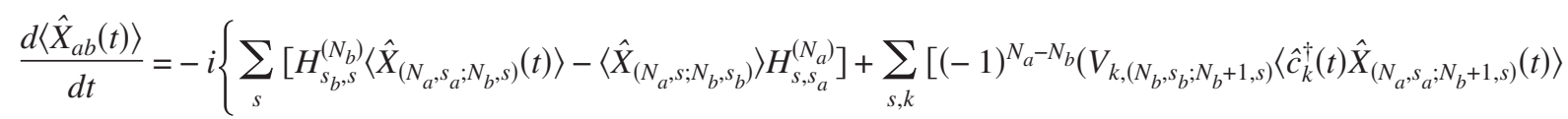

$$
\begin{aligned}
& \left.\left.\left.-V_{\left(N_{a}+1, s ; N_{a}, s_{a}\right), k}\left\langle\hat{X}_{\left(N_{b}, s_{b} ; N_{a}+1, s\right)}^{\dagger}(t) \hat{c}_{k}(t)\right\rangle\right)+V_{\left(N_{b}, s_{b} ; N_{b}-1, s\right), k}\left\langle\hat{X}_{\left(N_{b}-1, s ; N_{a}, s_{a}\right)}^{\dagger}(t) \hat{c}_{k}(t)\right\rangle-V_{k,\left(N_{a}-1, s ; N_{a}, s_{a}\right)}\left\langle\hat{c}_{k}^{\dagger}(t) \hat{X}_{\left(N_{a}-1, s ; N_{b}, s_{b}\right)}(t)\right\rangle\right]\right\},
\end{aligned}
$$


where $\Sigma_{s}$. is a sum over the molecular states inside each charge block, $\Sigma_{k} \ldots$ is a sum over the states in the contacts, and the factor $(-1)^{N_{a}-N_{b}}$ results from commuting $\hat{X}_{a b}$ with $\hat{c}_{k}$ $\left(\hat{c}_{k}^{\dagger}\right)$.

Correlation functions in the right of Eq. (A1) can be identified as lesser projections of the GFs [Eqs. (12) and (13)] defined on the Keldysh contour. Equations for these GFs are presented in Eqs. (14) and (15). Taking lesser projection of the equations of motion and applying the Langreth rules ${ }^{37}$ yield, e.g., for the first correlation function in Eq. (A1)

$$
\begin{aligned}
\left\langle\hat{c}_{k}^{\dagger}(t)\right. & \left.\hat{X}_{\left(N_{a}, s_{a} ; N_{b}+1, s\right)}(t)\right\rangle \\
\equiv & (-1)^{N_{a}-N_{b}-1} i G_{X c}(t, t) \\
= & (-1)^{N_{a}-N_{b}-1} \sum_{\mathcal{M}} V_{\overline{\mathcal{M}} k} \int_{-\infty}^{+\infty} d t_{1}\left[(-1)^{N_{a}-N_{b}-1}\right. \\
& \times\left\langle\hat{X}_{\mathcal{M}}^{\dagger}\left(t_{1}\right) \hat{X}_{\left(N_{a}, s_{a} ; N_{b}+1, s\right)}(t)\right\rangle g_{k}^{a}\left(t_{1}-t\right) \\
& +\theta\left(t-t_{1}\right)\left(\left\langle\hat{X}_{\left(N_{a}, s_{a} ; N_{b}+1, s\right)}(t) \hat{X}_{\mathcal{M}}^{\dagger}\left(t_{1}\right)\right\rangle\right. \\
& \left.\left.-(-1)^{N_{a}-N_{b}-1}\left\langle\hat{X}_{\mathcal{M}}^{\dagger}\left(t_{1}\right) \hat{X}_{\left(N_{a}, s_{a} ; N_{b}+1, s\right)}(t)\right\rangle\right) g_{k}^{<}\left(t_{1}-t\right)\right],
\end{aligned}
$$

where $g_{k}^{a,<}(t)$ are the advanced and lesser projections of the GF [Eq. (16)] and where the general property of GFs $G^{r}(t)$ $=\theta(t)\left[G^{>}(t)-G^{<}(t)\right]$ was used for the $G_{X X}$ GF. Once more, the factors $(-1)^{N_{a}-N_{b}-1}$ trace the Fermi or Bose character of $\hat{X}_{a b}$. Using $g_{k}^{a}(t)=\theta(-t)\left[g_{k}^{<}(t)-g_{k}^{>}(t)\right]$ and utilizing Eqs. (18) and (19) leads to final expression for the first correlation function in Eq. (A1). Repeating this consideration for the three other correlation functions in Eq. (A1) and using the resulting expressions in Eq. (A1), leads to Eq. (17).

\section{APPENDIX B: GREEN FUNCTIONS IN LIOUVILLE SPACE}

Here, we discuss the properties of the retarded and advanced GF in Liouville space. We start from the definitions in Eqs. (30) and (32). Using the property of the full unitary propagator

$$
\left\langle\left\langle\hat{A}\left|e^{-i \mathcal{L} t}\right| \hat{B}\right\rangle\right\rangle=\left\langle\left\langle\hat{A}^{\dagger}\left|e^{-i \mathcal{L} t}\right| \hat{B}^{\dagger}\right\rangle\right\rangle^{*},
$$

we find that

$$
\begin{aligned}
\left\langle\left\langle\hat{X}_{i j}\left|\mathcal{U}_{\mathrm{eff}}(t)\right| \hat{X}_{m n}\right\rangle\right\rangle & =\left\langle\left\langle\hat{X}_{j i}\left|\mathcal{U}_{\mathrm{eff}}(t)\right| \hat{X}_{n m}\right\rangle\right\rangle^{*} \\
& =\left\langle\left\langle\hat{X}_{n m}\left|\mathcal{U}_{\mathrm{eff}}^{\dagger}(t)\right| \hat{X}_{j i}\right\rangle\right\rangle,
\end{aligned}
$$

where the second equality comes from definition of the Hermitian conjugate. Using Eq. (B2) in Eq. (30), one gets

$$
\mathcal{G}_{i j, m n}^{a}(t)=\mathcal{G}_{m n, i j}^{r *}(-t)=i \theta(-t)\left\langle\left\langle\hat{X}_{m n}\left|\mathcal{U}_{\mathrm{eff}}(-t)\right| \hat{X}_{i j}\right\rangle\right\rangle .
$$

Note, that the definitions in Eqs. (30) and (31) lead to the usual Hermitian-type connection in Eq. (B3) between the retarded and the advanced GFs $\mathcal{G}^{a}=\left[\mathcal{G}^{r}\right]^{\dagger}$. An alternative definition could be

$$
\begin{aligned}
\mathcal{G}_{i j, m n}^{r}(t) & \equiv-i \theta(t)\left\langle\left\langle\hat{X}_{j i} \hat{I}_{K}\left|e^{-i \mathcal{L} t}\right| \hat{X}_{m n} \hat{\rho}_{K}^{e q}\right\rangle\right\rangle \\
& =-i \theta(t)\left\langle\left\langle\hat{X}_{j i}\left|\mathcal{U}_{\mathrm{eff}}(t)\right| \hat{X}_{m n}\right\rangle\right\rangle, \\
\mathcal{G}_{i j, m n}^{a}(t) & \equiv i \theta(-t)\left\langle\left\langle\hat{X}_{n m} \hat{I}_{K}\left|e^{i \mathcal{L} t}\right| \hat{X}_{i j} \hat{\rho}_{K}^{e q}\right\rangle\right\rangle \\
& =i \theta(-t)\left\langle\left\langle\hat{X}_{j i}\left|\mathcal{U}_{\mathrm{eff}}^{\dagger}(-t)\right| \hat{X}_{m n}\right\rangle\right\rangle,
\end{aligned}
$$

and would lead to Liouvillian conjugation ${ }^{44} \mathcal{G}^{a}=\left[\mathcal{G}^{r}\right]^{\times}$or

$$
\mathcal{G}_{i j, m n}^{a}(t)=\mathcal{G}_{n m, j i}^{r *}(-t)
$$

This alternative definition will not be used in this paper.

\section{APPENDIX C: EXPRESSION FOR $\mathcal{L}_{\text {eff }}$}

We start from Eqs. (37) and (38) and use the free propagator instead of the effective one. This leads to

$$
\begin{aligned}
\mathcal{G}_{i j, m n}^{(0) r}(t) & =-i \theta(t)\left\langle\left\langle\hat{X}_{j i} \mid e^{-i \mathcal{L}_{M^{t}} \mid} \hat{X}_{n m}\right\rangle\right\rangle \\
& \equiv-i \theta(t)\left\langle j\left|e^{-i \hat{H}_{M^{t}}}\right| n\right\rangle\left\langle m\left|e^{i \hat{H}_{M^{t}}}\right| i\right\rangle,
\end{aligned}
$$

$$
\begin{aligned}
\mathcal{G}_{i j, m n}^{(0) a}(t) & =i \theta(-t)\left\langle\left\langle\hat{X}_{j i} \mid e^{-i \mathcal{L}_{M^{\dagger}}^{\dagger} \mid} \hat{X}_{n m}\right\rangle\right\rangle \\
& \equiv i \theta(-t)\left\langle j\left|e^{-i \hat{H}_{M^{t}}}\right| n\right\rangle\left\langle m\left|e^{i \hat{H}_{m} t}\right| i\right\rangle .
\end{aligned}
$$

Substituting Eqs. (C1) and (C2) into Eq. (35), and using the Markov approximation

$$
\rho_{a b}\left(t_{1}\right) \approx \sum_{c, d}\left\langle\left\langle a b\left|e^{i \mathcal{L}_{M}\left(t-t_{1}\right)}\right| c d\right\rangle\right\rangle \rho_{c d}(t),
$$

one gets the (Markovian) Redfield QME

$$
\frac{d \rho_{a b}(t)}{d t}=-i \sum_{c, d}\left\langle\left\langle a b\left|\mathcal{L}_{\mathrm{eff}}\right| c d\right\rangle\right\rangle \rho_{c d}(t),
$$

where the generator of our model takes the form 


$$
\begin{aligned}
& -i \mathcal{L}_{(a ; b),(c ; d)}^{\mathrm{eff}}=i \mathcal{L}_{(b ; a),(d ; c)}^{\mathrm{eff} \dagger}=-i\left\{\delta_{N_{a}, N_{c}} \delta_{N_{b}, N_{d}}\left[H_{s_{a}, s}^{\left(N_{a}\right)} \delta_{s_{b}, s_{d}}-\delta_{s_{a}, s_{c}} H_{s_{d}, s_{b}}^{\left(N_{b}\right)}\right]-\frac{1}{2} \sum_{i, j} \sum_{p, r}\right. \\
& \times\left[\delta_{N_{a}+1, N_{c}} \delta_{N_{b}+1, N_{d}}(-1)^{N_{a}-N_{b}} \times\left(U_{r i}^{\left(N_{a}+1\right)} \stackrel{*}{U_{s_{c} i}^{\left(N_{a}+1\right)}} U_{s_{a} j}^{\left(N_{a}\right)} \stackrel{*}{U}_{p j}^{\left(N_{a}\right)} \Sigma_{\left(N_{b}, s_{b} ; N_{b}+1, s_{d}\right),\left(N_{a}, p ; N_{a}+1, r\right)}^{>}\left(E_{i}^{\left(N_{a}+1\right)}-E_{j}^{\left(N_{a}\right)}\right)\right.\right.
\end{aligned}
$$

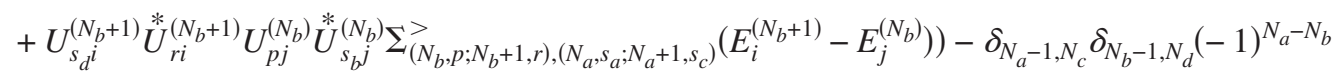

$$
\begin{aligned}
& \times\left(U_{s_{a} i}^{\left(N_{a}\right)} \stackrel{*}{U_{r i}^{\left(N_{a}\right)}} U_{p j}^{\left(N_{a}-1\right)} \stackrel{*}{U}_{s_{c} j}^{\left(N_{a}-1\right)} \Sigma_{\left(N_{a}-1, p ; N_{a}, r\right),\left(N_{b}-1, s_{d} ; N_{b}, s_{b}\right)}^{<}\left(E_{i}^{\left(N_{a}\right)}-E_{j}^{\left(N_{a}-1\right)}\right)\right. \\
& \left.+U_{r i}^{\left(N_{b}\right)} \stackrel{*}{U}_{s_{b} i}^{\left(N_{b}\right)} U_{s_{d} j}^{\left(N_{b}-1\right)} U_{p j}^{*}\left(N_{b}-1\right) \Sigma_{\left(N_{a}-1, s_{c} ; N_{a}, s_{a}\right),\left(N_{b}-1, p ; N_{b}, r\right)}^{<}\left(E_{i}^{\left(N_{b}\right)}-E_{j}^{\left(N_{b}-1\right)}\right)\right) \\
& +\delta_{N_{a}, N_{c}} \delta_{N_{b}, N_{d}} \delta_{s_{a}, s_{c}} \sum_{s}\left(U_{r i}^{\left(N_{b}+1\right)} \stackrel{*}{U}_{s i}^{\left(N_{b}+1\right)} U_{s_{d} j}^{\left(N_{b}\right)} \stackrel{*}{U j}_{p j}^{\left(N_{b}\right)} \Sigma_{\left(N_{b}, s_{b} ; N_{b}+1, s\right),\left(N_{b}, p ; N_{b}+1, r\right)}^{<}\left(E_{i}^{\left(N_{b}+1\right)}-E_{j}^{\left(N_{b}\right)}\right)\right. \\
& \left.-U_{s_{d} i}^{\left(N_{b}\right)} \stackrel{*}{U_{r i}^{\left(N_{b}\right)}} U_{p j}^{\left(N_{b}-1\right)} \stackrel{*}{U}_{s j}^{\left(N_{b}-1\right)} \Sigma_{\left(N_{b}-1, p ; N_{b}, r\right),\left(N_{b}-1, s ; N_{b}, s_{b}\right)}^{>}\left(E_{i}^{\left(N_{b}\right)}-E_{j}^{\left(N_{b}-1\right)}\right)\right)
\end{aligned}
$$

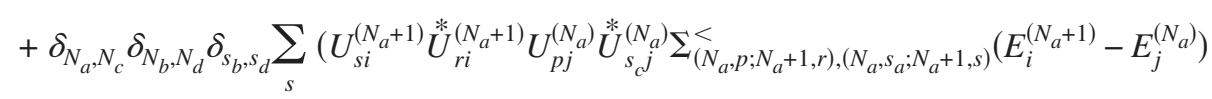

$$
\begin{aligned}
& \left.\left.\left.-U_{r i}^{\left(N_{a}\right)} \stackrel{*}{U}_{s_{c} i}^{\left(N_{a}\right)} U_{s j}^{\left(N_{a}-1\right)} \stackrel{*}{U}_{p j}^{\left(N_{a}-1\right)} \Sigma_{\left(N_{a}-1, s ; N_{a}, s_{a}\right),\left(N_{a}-1, p ; N_{a}, r\right)}^{>}\left(E_{i}^{\left(N_{a}\right)}-E_{j}^{\left(N_{a}-1\right)}\right)\right)\right]\right\},
\end{aligned}
$$

where $\mathbf{U}^{(N)}$ are the unitary transformations diagonalizing the charge blocks $\mathbf{H}_{M}^{(N)}$ of molecular Hamiltonian $(7)$, and $E_{i}^{(N)}$ are corresponding eigenvalues.

*Present address: Center for Nonlinear Phenomena and Complex Systems, Université Libre de Bruxelles, Code Postal 231, Campus Plaine, B-1050 Brussels, Belgium.

${ }^{1}$ M. Galperin, M. A. Ratner, A. Nitzan, and A. Troisi, Science 319, 1056 (2008).

${ }^{2}$ J. B. Neaton, M. S. Hybertsen, and S. G. Louie, Phys. Rev. Lett. 97, 216405 (2006).

${ }^{3}$ S. Y. Quek, J. B. Neaton, M. S. Hybertsen, E. Kaxiras, and S. G. Louie, Phys. Rev. Lett. 98, 066807 (2007).

${ }^{4}$ K. S. Thygesen, Phys. Rev. Lett. 100, 166804 (2008).

${ }^{5}$ K. S. Thygesen and A. Rubio, Phys. Rev. Lett. 102, 046802 (2009).

${ }^{6}$ D. R. Ward, N. J. Halas, J. W. Ciszek, J. M. Tour, Y. Wu, P. Nordlander, and D. Natelson, Nano Lett. 8, 919 (2008).

${ }^{7}$ J. Bonca and S. A. Trugman, Phys. Rev. Lett. 75, 2566 (1995).

${ }^{8}$ B. Muralidharan, A. W. Ghosh, and S. Datta, Phys. Rev. B 73, 155410 (2006).

${ }^{9}$ L. Siddiqui, A. W. Ghosh, and S. Datta, Phys. Rev. B 76, 085433 (2007).

${ }^{10}$ J. Koch and F. von Oppen, Phys. Rev. Lett. 94, 206804 (2005).

${ }^{11}$ J. Koch, F. von Oppen, and A. V. Andreev, Phys. Rev. B 74, 205438 (2006).

${ }^{12}$ J. Koch, M. E. Raikh, and F. von Oppen, Phys. Rev. Lett. 96, 056803 (2006).

${ }^{13}$ J. Koch, E. Sela, Y. Oreg, and F. von Oppen, Phys. Rev. B 75, 195402 (2007).

${ }^{14}$ C. Timm, Phys. Rev. B 77, 195416 (2008).

${ }^{15}$ E. G. Petrov, V. May, and P. Hänggi, Chem. Phys. 319, 380 (2005).

${ }^{16}$ E. G. Petrov, V. May, and P. Hänggi, Phys. Rev. B 73, 045408
(2006).

${ }^{17}$ H. Schoeller, Lect. Notes Phys. 544, 137 (2000).

${ }^{18}$ J. N. Pedersen and A. Wacker, Phys. Rev. B 72, 195330 (2005).

${ }^{19}$ X. Q. Li, J. Luo, Y. G. Yang, P. Cui, and Y. J. Yan, Phys. Rev. B 71, 205304 (2005).

${ }^{20}$ S. Welack, M. Schreiber, and U. KleinekathÃferb, J. Chem. Phys. 124, 044712 (2006).

${ }^{21}$ U. Harbola, M. Esposito, and S. Mukamel, Phys. Rev. B 74, 235309 (2006).

${ }^{22}$ I. Sandalov, B. Johansson, and O. Eriksson, Int. J. Quantum Chem. 94, 113 (2003).

${ }^{23}$ J. Fransson, Phys. Rev. B 72, 075314 (2005).

${ }^{24}$ M. Galperin, A. Nitzan, and M. A. Ratner, Phys. Rev. B 78, 125320 (2008).

${ }^{25}$ A. Mitra, I. Aleiner, and A. J. Millis, Phys. Rev. B 69, 245302 (2004).

${ }^{26}$ M. Galperin, A. Nitzan, and M. A. Ratner, Phys. Rev. B 73, 045314 (2006).

${ }^{27}$ See footnote [51] in Ref. 24.

${ }^{28}$ J. N. Pedersen, D. Bohr, A. Wacker, T. Novotný, P. Schmitteckert, and K. Flensberg, Phys. Rev. B 79, 125403 (2009).

${ }^{29}$ M. Galperin and S. Tretiak, J. Chem. Phys. 128, 124705 (2008).

${ }^{30}$ I. V. Ovchinnikov and D. Neuhauser, J. Chem. Phys. 122, 024707 (2005).

${ }^{31}$ S. Datta, arXiv:cond-mat/0603034 (unpublished).

${ }^{32} \mathrm{H}$. Haug and A. Jauho, Quantum Kinetics in Transport and $\mathrm{Op}$ tics of Semiconductors (Springer-Verlag, Berlin, 1996).

${ }^{33}$ J. Fransson, O. Eriksson, and I. Sandalov, Phys. Rev. B 66, 195319 (2002).

${ }^{34}$ Y. Meir and N. S. Wingreen, Phys. Rev. Lett. 68, 2512 (1992). 
${ }^{35}$ A. P. Jauho, N. S. Wingreen, and Y. Meir, Phys. Rev. B 50, 5528 (1994).

${ }^{36}$ M. Galperin, A. Nitzan, and M. A. Ratner, Phys. Rev. B 75, 155312 (2007).

${ }^{37}$ D. C. Langreth, in Linear and Nonlinear Electron Transport in Solids, edited by J. T. Devreese and D. E. Doren (Plenum, New York, 1976), pp. 3-32.

${ }^{38}$ H.-P. Breuer and F. Petruccione, The Theory of Open Quantum Systems (Oxford University Press, Oxford, 2002).

${ }^{39}$ C. W. Gardiner and P. Zoller, Quantum Noise, 2nd ed. (SpingerVerlag, Berlin, 2000)
${ }^{40}$ R. Kubo, M. Toda, and N. Hashitsume, Statistical Physics II: Nonequilibrium Statistical Mechanics, 2nd ed. (Springer-Verlag, Berlin, 1998).

${ }^{41}$ H. J. Carmichael, Statistical Methods in Quantum Optics (Springer-Verlag, Berlin, 1999), Vol. 1.

${ }^{42}$ G. D. Mahan, Many-Particle Physics, 3rd ed. (Kluwer, New York/Academic, New York/Plenum, New York, 2000).

${ }^{43}$ M. Galperin, A. Nitzan, and M. A. Ratner, Phys. Rev. B 76, 035301 (2007).

${ }^{44}$ A. Ben-Reuven and S. Mukamel, J. Phys. A 8, 1313 (1975). 